YITP-SB-07-01

\title{
On Semiclassical Limits of String States
}

\author{
Jose J. Blanco-Pillado ${ }^{a, 1}$, Alberto Iglesias ${ }^{b, 2}$ and Warren Siegel ${ }^{c, 3}$ \\ ' Institute of Cosmology, Department of Physics and Astronomy, Tufts University, Medford, MA 02155 \\ ${ }^{b}$ Department of Physics, University of California, Davis, CA 95616 \\ ${ }^{c}$ C. N. Yang Institute for Theoretical Physics, State University of New York, Stony Brook, NY 11794
}

\begin{abstract}
We explore the relation between classical and quantum states in both open and closed (super)strings discussing the relevance of coherent states as a semiclassical approximation. For the closed string sector a gauge-fixing of the residual world-sheet rigid translation symmetry of the light-cone gauge is needed for the construction to be possible. The circular target-space loop example is worked out explicitly.
\end{abstract}

\footnotetext{
${ }^{1}$ E-mail: jose@cosmos.phy.tufts.edu

${ }^{2}$ E-mail: iglesias@physics.ucdavis.edu

${ }^{3}$ E-mail: siegel@insti.physics.sunysb.edu
} 


\section{Introduction}

The motivation to investigate the semiclassical limit of fundamental strings is twofold. First, in view of the revived interest in the possibility of producing superstrings of cosmic size in models of brane inflation, it has been suggested that brane annihilation would leave behind a network of lower dimensional extended objects $[1,2,3,4]$ which would be seen as strings from the four dimensional point of view. This realization opens up the possibility of observing the cosmological consequences of cosmic strings, either from fundamental strings or from (wrapped) D-branes. It is therefore interesting to understand how to reconcile the usual quantum mechanical treatment of fundamental strings with their expected classical behaviour at cosmological scales. Second, the semiclassical limit discussed in this paper may play a role in relation to the possible microscopic counting of states $[5,6,7,8]$ associated with known classical supergravity solutions.

It is usually assumed that the description of a semiclassical string state (a string of macroscopic size) is in terms of a coherent superposition of the fundamental string quanta. However, ealier attempts to build this type of state in the covariant gauge quantization face serious difficulties [9]. In the next section we motivate the use of the lightcone gauge coherent state to give an accurate microscopic description of extended open string solutions (spinning string configuration). We contrast the result with the alternative mass eigenstates ("perturbative states") with the same angular momentum, emphasizing the advantages of the former. In section 3 we focus on the closed string case. We describe the obstacle to the naive extrapolation from the open string case. We then provide the solution, suitable for the case of a circular target space loop, by gauge-fixing the residual rigid $\sigma$ translation symmetry. This is done in three ways: in unitary gauge, through a BRST method and in Gupta-Bleuler like quantization.

\section{Open strings}

In this section we explore the relation between perturbative quantum states and their classical counterparts for open strings. In particular, we will find the closest quantum state to the leading classical Regge trajectory that corresponds to the following spinning configuration,

$$
\begin{aligned}
X^{0} & =A \tau, \\
X^{1} & =A \sin \tau \cos \sigma, \\
X^{2} & =A \cos \tau \cos \sigma .
\end{aligned}
$$

In the conventions of [10], the general solution for the open strings in light-cone gauge is given by the following expressions,

$$
\begin{aligned}
X^{+} & =x^{+}+l^{2} p^{+} \tau, \\
X^{i} & =x^{i}+l^{2} p^{i} \tau+i l \sum_{n \neq 0} \frac{1}{n} \alpha_{n}^{i} \mathrm{e}^{-i n \tau} \cos n \sigma,
\end{aligned}
$$




$$
X^{-}=x^{-}+l^{2} p^{-} \tau+i l \sum_{n \neq 0} \frac{1}{n} \alpha_{n}^{-} \mathrm{e}^{-i n \tau} \cos n \sigma
$$

On the other hand, in order to fulfill the constraints the $\alpha_{n}^{-}$are restricted to be functions of the physical transverse directions,

$$
\alpha_{n}^{-}=\frac{1}{2 l p^{+}}\left(\sum_{m=-\infty}^{\infty}: \alpha_{n-m}^{i} \alpha_{m}^{i}:-2 a \delta_{n}\right)
$$

where the $a$ coefficcient comes from the normal ordering of the $\alpha$ operators. Furthermore we can also write the mass and the angular momentum in terms of transverse modes in the following way,

$$
\begin{aligned}
M^{2} & =\frac{2}{l^{2}}\left(\sum_{n=1}^{\infty} \alpha_{-n}^{i} \alpha_{n}^{i}-a\right) \\
J^{i j} & =-i \sum_{n=1}^{\infty} \frac{1}{n}\left(\alpha_{-n}^{i} \alpha_{n}^{j}-\alpha_{-n}^{j} \alpha_{n}^{i}\right) .
\end{aligned}
$$

\subsection{Classical Regge trajectory in the light-cone gauge}

We start out by finding the description in the lightcone gauge of the classical solution of the open string found in (1). It is clear that this state corresponds to a string spinning around its center of mass, which has been set to the origin of the coordinates, which implies that,

$$
x^{+}=x^{-}=x^{i}=p^{i}=0 .
$$

Also, it is easy to see that the only excited oscillators in this solution are,:

$$
\begin{aligned}
\alpha_{1}^{1} & =\left(\alpha_{-1}^{1}\right)^{*}=-\frac{i A}{2 l}, \\
\alpha_{1}^{2} & =\left(\alpha_{-1}^{2}\right)^{*}=\frac{A}{2 l} .
\end{aligned}
$$

Which in turn implies that,

$$
\begin{aligned}
& X^{1}=i l\left(-\alpha_{-1}^{1} e^{i \tau}+\alpha_{1}^{1} e^{-i \tau}\right) \cos \sigma=A \cos \sigma \cos \tau, \\
& X^{2}=i l\left(-\alpha_{-1}^{2} e^{i \tau}+\alpha_{1}^{2} e^{-i \tau}\right) \cos \sigma=A \cos \sigma \sin \tau .
\end{aligned}
$$

And for the $(-)$ oscillators we obtain,

$$
\alpha_{0}^{-}=l p^{-}=\frac{1}{2 l p^{+}}\left(\frac{A}{l}\right)^{2},
$$

with all the other $\alpha_{n}^{-}=0$. Note that the only non-trivial cases are $n= \pm 2$, which in our case are still zero,

$$
\alpha_{2}^{-}=\frac{1}{2 l p^{+}}\left(\alpha_{1}^{1} \alpha_{1}^{1}+\alpha_{1}^{2} \alpha_{1}^{2}\right)=\frac{1}{2 l p^{+}}\left[-\left(\frac{A}{2 l}\right)^{2}+\left(\frac{A}{2 l}\right)^{2}\right]=0,
$$


and the same for $\alpha_{-2}^{-}$. If we want the string to move on the $1-2$ plane only, we have to impose that $X^{9}=0$,

$$
X^{9}=\frac{1}{\sqrt{2}}\left(X^{+}-X^{-}\right)=\frac{1}{\sqrt{2}}\left(l^{2} p^{+}-l^{2} p^{-}\right) \tau=0
$$

in other words, that $p^{+}=p^{-}=A / \sqrt{2} l^{2}$. This also means that,

$$
X^{0}=\frac{1}{\sqrt{2}}\left(X^{+}+X^{-}\right)=\frac{1}{\sqrt{2}}\left(l^{2} p^{+}+l^{2} p^{-}\right) \tau=A \tau .
$$

So, putting all these results together, we finally get a solution of the form (1),

$$
\begin{aligned}
& X^{0}=A \tau, \\
& X^{1}=A \sin \tau \cos \sigma, \\
& X^{2}=A \cos \tau \cos \sigma .
\end{aligned}
$$

We can also use the equations given above to compute, in the classical limit, the observables of this state, i.e., its mass and angular momentum.

$$
\begin{aligned}
M^{2} & =\frac{2}{l^{2}}\left(\sum_{n=1}^{\infty} \alpha_{-n}^{i} \alpha_{n}^{i}\right)=\frac{2}{l^{2}}\left(\alpha_{-1}^{1} \alpha_{1}^{1}+\alpha_{-1}^{2} \alpha_{1}^{2}\right)=\frac{A^{2}}{l^{4}} \\
J^{12} & =-i\left(\alpha_{-1}^{1} \alpha_{1}^{2}-\alpha_{-1}^{2} \alpha_{1}^{1}\right)=\frac{A^{2}}{2 l^{2}}
\end{aligned}
$$

which indeed show that these configurations belong to the classical Regge trajectory of maximum angular momentum per unit mass.

\subsection{Quantum State}

We will now try to obtain the quantum state for the open bosonic string that resembles the classical case described above. There seem to be two natural possibilities:

\subsection{1 "Perturbative" Regge State (Mass Eigenstates)}

The classical calculation suggests that we construct the quantum state for this solution in the following way,

$$
|\psi\rangle=\frac{1}{\sqrt{2^{n} n !}}\left(\alpha_{-1}^{2}-i \alpha_{-1}^{1}\right)^{n}|0\rangle .
$$

The reason to choose this particular configuration becomes clear when we realize that this state is, in fact, an eigenstate of mass and angular momentum,

$$
\begin{aligned}
M^{2}|\psi\rangle & =\frac{2}{l^{2}}\left(\alpha_{-1}^{1} \alpha_{1}^{1}+\alpha_{-1}^{2} \alpha_{1}^{2}-1\right)|\psi\rangle=\frac{2}{l^{2}}(n-1)|\psi\rangle, \\
J^{12}|\psi\rangle & =-i\left(\alpha_{-1}^{1} \alpha_{1}^{2}-\alpha_{-1}^{2} \alpha_{1}^{1}\right)|\psi\rangle=n|\psi\rangle .
\end{aligned}
$$


Using the identification $n=1+A^{2} / 2 l^{2}$, we see that this state has identical values of mass and angular momentum to the classical configuration in the $n \gg 1$ or $A \gg l$ limit. In fact, they saturate the quantum inequality for mass eigenstates given by,

$$
J^{12} \leq \frac{l^{2}}{2} M^{2}+1
$$

On the other hand, this is not an eigenstate of the position of the string. It is easy to show that, in this state, the expectation value of the spatial part of the string position operator is equal to zero for all values of $\sigma$ and $\tau$, namely,

$$
\begin{aligned}
\left\langle\psi\left|\alpha_{n}^{i}\right| \psi\right\rangle & =0 \\
\left\langle\psi\left|\alpha_{k}^{-}\right| \psi\right\rangle & =\left\langle\psi\left|\frac{1}{2 l p^{+}} \sum_{m=-\infty}^{\infty} \alpha_{k-m}^{i} \alpha_{m}^{i}\right| \psi\right\rangle=0,
\end{aligned}
$$

for $k \neq 0$. Finally,

$$
\left\langle\psi\left|\alpha_{0}^{-}\right| \psi\right\rangle=\frac{n-1}{l p^{+}}
$$

which implies that ${ }^{4}$

$$
\begin{aligned}
\left\langle\psi\left|X^{0}\right| \psi\right\rangle & =A \tau, \\
\left\langle\psi\left|X^{i}\right| \psi\right\rangle & =0, \\
\left\langle\psi\left|X^{9}\right| \psi\right\rangle & =0 .
\end{aligned}
$$

We notice that while it is true that this state has similar properties to the classical one, it clearly does not resemble the macroscopic string state, in the sense that its spacetime motion is not reproduced at all, not even taking a high excitation number (i.e., $n \gg 1$ does not produce a semiclassical limit).

\subsection{2 "Coherent" Regge State}

On the other hand, it seems more reasonable to try to mimic the classical configuration by constructing a coherent state of the form,

$$
|\phi\rangle=e^{v \alpha_{-1}^{1}-v^{*} \alpha_{1}^{1}} e^{-i v \alpha_{-1}^{2}-i v^{*} \alpha_{1}^{2}}|0\rangle,
$$

where $v$ is a parameter related to $p^{+},\left(p^{+}=l^{-1} \sqrt{2|v|^{2}-1}\right)$ and to the amplitude of the spacetime oscillations.

\footnotetext{
${ }^{4}$ Where we have used the relations noted before between the different sets of parameters, $p^{+}=\frac{A}{\sqrt{2} l^{2}}$ as well as $n=\frac{A^{2}}{2 l^{2}}+1$.
} 
This state has the following expectation values for the energy and angular momentum,

$$
\begin{aligned}
\left\langle\phi\left|M^{2}\right| \phi\right\rangle & =\frac{2}{l^{2}}\left\langle\phi\left|\left(\sum_{n=1}^{\infty} \alpha_{-n}^{i} \alpha_{n}^{i}-1\right)\right| \phi\right\rangle \\
& =\frac{2}{l^{2}}\left\langle\phi\left|\left(\alpha_{-1}^{1} \alpha_{1}^{1}+\alpha_{-1}^{2} \alpha_{1}^{2}-1\right)\right| \phi\right\rangle=\frac{2}{l^{2}}\left(2|v|^{2}-1\right), \\
\left\langle\phi\left|J^{12}\right| \phi\right\rangle & =-i\left\langle\phi\left|\left(\alpha_{-1}^{1} \alpha_{1}^{2}-\alpha_{-1}^{2} \alpha_{1}^{1}\right)\right| \phi\right\rangle=2|v|^{2}
\end{aligned}
$$

which also correspond to the values obtained in the previous section by considering the identification $2|v|^{2}=n$. The key point, however, is that this state does have the spacetime position expectation value of an extended string, namely,

$$
\begin{aligned}
\left\langle\phi\left|X^{0}\right| \phi\right\rangle & =l \tau \sqrt{4|v|^{2}-2}=A \tau, \\
\left\langle\phi\left|X^{1}\right| \phi\right\rangle & =2 l v \sin \tau \cos \sigma=\sqrt{A^{2}+2 l^{2}} \sin \tau \cos \sigma, \\
\left\langle\phi\left|X^{2}\right| \phi\right\rangle & =2 l v \cos \tau \cos \sigma=\sqrt{A^{2}+2 l^{2}} \cos \tau \cos \sigma, \\
\left\langle\phi\left|X^{9}\right| \phi\right\rangle & =0,
\end{aligned}
$$

which for $A \gg l$ approaches the classical solution discussed above. ${ }^{5}$ This shows that the coherent state is a much closer match to the classical solution than the previously considered construction.

\section{Closed strings}

The calculations in the previous section show how one can obtain a semiclassical coherent state for open strings in a very similar way to the simple harmonic oscillator. However, no such construction is available for closed strings, except in the approach of "semiclassical" quantization (in the sense of [11], where the constraints are satisfied in mean value) as in [12]. In this section we will present the obstacle in finding a macroscopic state from perturbative closed string states in the lightcone gauge, and propose a solution.

\subsection{The problem of a microscopic perturbative description}

Consider the first order form of of the bosonic string action with world-sheet coordinates $m=(0,1) \equiv(\sigma, \tau)$

$$
S=\frac{1}{2 \pi \alpha^{\prime}} \int d^{2} \sigma\left(\partial_{m} X \cdot P^{m}+g_{m n} \frac{1}{2} P^{m} \cdot P^{n}\right)
$$

where $g_{m n}=(-h)^{-1 / 2} h_{m n}$ is the unit determinant part of the world-sheet metric $h_{m n}$ (related to the second order form [13] using $\left.P^{m}=(-h)^{1 / 2} h^{m n} \partial_{n} X\right)$.

\footnotetext{
${ }^{5}$ Note that in this limit we also recover the same values for the mass and angular momentum of the classical configuration.
} 
Integrating out $P^{\sigma}$ via its equation of motion:

$$
P^{\sigma}=-\frac{1}{g_{11}}\left(X^{\prime}+g_{01} P^{\tau}\right)
$$

the action becomes

$$
S=\frac{1}{2 \pi \alpha^{\prime}} \int d^{2} \sigma\left[\dot{X} \cdot P-\frac{1}{2 g_{11}}\left(X^{\prime 2}+P^{2}\right)-\frac{g_{01}}{g_{11}} X^{\prime} \cdot P\right],
$$

where $P^{\tau} \equiv P$ to simplify notation. The reparametrization symmetry of (35) can be used to set $g_{11}=1$ and $g_{01}=0$. Further, Weyl symmetry can be used to set $h_{11}=1$ and residual semilocal symmetry to set

$$
\left.X^{+}=\tau, \quad P_{+}=1 \quad \text { (light }- \text { cone gauge }\right) .
$$

Let us now look closely at the constraint obtained by varying $g_{01}$, i.e., $X^{\prime} \cdot P=0$ that has the following mode decomposition $\left(\int_{0}^{\pi} d \tau \mathrm{e}^{2 i m \tau} \cdots\right)$ :

$$
C_{m}=\alpha_{m}^{-}-\widetilde{\alpha}_{m}^{-}+\sum_{n \neq 0}\left(\alpha_{m-n}^{i} \alpha_{n}^{i}-\widetilde{\alpha}_{m-n}^{i} \widetilde{\alpha}_{n}^{i}\right)
$$

where

$$
C_{0}=\Delta N, \quad\left(\text { recall } \alpha_{0}^{-}=\widetilde{\alpha}_{0}^{-}\right)
$$

is the generator of rigid $\sigma$ shifts $\left(\delta X=\epsilon \partial_{\sigma} X\right.$, with constant $\left.\epsilon\right)$. Upon quantization, if a physical state $\mid$ phys $\rangle$ satisfies the constraint $C_{0}$ :

$$
\Delta N \mid \text { phys }\rangle=0
$$

then, it follows that

$$
0 \equiv\langle\text { phys }|[\Delta N, X]| \text { phys }\rangle=\partial_{\sigma}\langle X\rangle
$$

This shows that the string would appear to be stuck at a fixed point $\langle X\rangle$ for all $\sigma$, making it impossible, in this gauge, to have a macroscopic extended closed string. The reason for this is that we have not fixed the gauge completely so, in practice, we are integrating over all the gauges compatible with the lightcone gauge which, of course, yields the aforementioned center of mass position for the whole string. This makes the operator $X$ not the right quantity to look at in this gauge if we are interested in evaluating the semiclassical position of the string. The way out of this problem that we suggest in the following section is to fix the gauge completely before evaluating the position of the string. 


\subsection{Gauge-fixing $\sigma$ translations}

In this subsection we will fix the gauge for the residual rigid reparametrization symmetry $\sigma \rightarrow \sigma+\epsilon$ that remains after choosing light-cone gauge for closed strings. We do this by prescribing the value of one of the coordinates modes of the string. This fixes the symmetry in a way similar to the way in which the open string arises from the closed string by removing the modes of one handedness [14].

Our goal here will be that of describing a circular string. Once we are in the lightcone gauge, $X^{+}=\left(X^{0}+X^{9}\right) / \sqrt{2} \propto \tau$, we single out two of the coordinates (that span the plane in which the circular loop lies): $X^{1}$ and $X^{2}$. The solutions for the equations of motion of these coordinates have the usual decomposition into left and right moving modes, namely, $X^{i}=X_{L}^{i}+X_{R}^{i}{ }^{6}$

We propose the following additional gauge-fixing condition suitable for the description of the circular string loop states:

$$
\Phi=\frac{1}{\pi} \int d \sigma \mathrm{e}^{-2 i \sigma}\left(\partial_{-} X^{1}-v l \mathrm{e}^{-2 i(\tau-\sigma)}\right)=0
$$

where $\partial_{-}=\partial_{\tau}-\partial_{\sigma}$ and the parameter $v$ will be related to the radius of the circle. Note that this is only a condition involving the left-moving part of $X^{1}$ since $\partial_{-} X_{R} \equiv 0$.

To the action (37) we add the gauge-fixing term:

$$
\mathcal{L}_{g f}=\lambda \Phi
$$

such that $\lambda$ acts as the Lagrange multiplier enforcing the gauge conditions $\Phi=0$ (i.e., determining $\left.\alpha_{1}^{1}\right)$.

After reaching light-cone gauge, the only remaining piece of the third term in the action (37) is given by

$$
\int d \tau g_{0} \sum_{n}\left(\alpha_{-n}^{i}(\tau) \alpha_{n}^{i}(\tau)-\widetilde{\alpha}_{-n}^{i}(\tau) \widetilde{\alpha}_{n}^{i}(\tau)\right)
$$

where $g_{0}$ stands for the zero mode of $g_{01}$, we have used the decomposition $\partial_{-} X^{1}=$ $\sum \alpha_{n}^{1}(\tau) \mathrm{e}^{2 n i \sigma}, \ldots$ etc.

Varying with respect to $\lambda$ and $g_{0}$ we obtain the gauge-fixing condition and the constraint that can be solved (if $v \neq 0$ ) for $\alpha_{1}^{1}(\tau)$ and $\alpha_{-1}^{1}(\tau)$ respectively. On the solutions,

${ }^{6}$ For closed strings the standard decomposition into left and right-movers is

$$
\begin{aligned}
X^{i} & =X_{L}^{i}+X_{R}^{i}, \\
X_{L}^{i} & =\frac{1}{2} x^{i}+\frac{1}{2} l^{2} p^{i}(\tau-\sigma)+\frac{i}{2} l \sum_{n \neq 0} \frac{1}{n} \alpha_{n}^{i} \mathrm{e}^{-2 i n(\tau-\sigma)}, \\
X_{R}^{i} & =\frac{1}{2} x^{i}+\frac{1}{2} l^{2} p^{i}(\tau+\sigma)+\frac{i}{2} l \sum_{n \neq 0} \frac{1}{n} \tilde{\alpha}_{n}^{i} \mathrm{e}^{-2 i n(\tau+\sigma)},
\end{aligned}
$$

where $i=1, \cdots, 8$, and $x^{i}$ and $p^{i}$ are the center of mass position and momentum of the loop. 
$\alpha_{n}(\tau)=\alpha_{n} \mathrm{e}^{-2 i n \tau}, \ldots$, etc. Therefore, we obtain,

$$
\begin{aligned}
\alpha_{1}^{1} & =v \\
\alpha_{-1}^{1} & =-\frac{1}{v}\left(\sum_{m \geq 2} \alpha_{-m}^{1} \alpha_{m}^{1}+\sum_{n \geq 1} \alpha_{-n}^{j} \alpha_{n}^{j}-\widetilde{\alpha}_{-n}^{i} \widetilde{\alpha}_{n}^{i}\right),
\end{aligned}
$$

where $j=2, \cdots, 8$. These results show that the idea behind our gauge fixing choice in (46) is very much like the one used to solve the constraints in the lightcone gauge expressing $\alpha_{n}^{-}$in terms of the transverse modes.

In this gauge, then, the mode decomposition of $X_{L}^{1}$ is different from the usual. It reads,

$$
\begin{aligned}
X_{L}^{1}= & \frac{1}{2} x^{i}+\frac{1}{2} l^{2} p^{i}(\tau-\sigma)-\frac{i}{2} l \alpha_{-1}^{1} \mathrm{e}^{2 i(\tau-\sigma)}+\frac{i}{2} v l \mathrm{e}^{-2 i(\tau-\sigma)} \\
& +\frac{i}{2} l \sum_{n>1} \frac{1}{n}\left(\alpha_{n}^{1} \mathrm{e}^{-2 i n(\tau-\sigma)}-\alpha_{-n}^{1} \mathrm{e}^{2 i n(\tau-\sigma)}\right),
\end{aligned}
$$

where $\alpha_{-1}^{1}$ should be interpreted as the operator on the rhs of (50).

Alternatively, using the BRST method, the same result can be obtained with a gaugefixing term linear in $\Phi$. The BRST charge in this case contains an extra term:

$$
Q_{\text {extra }}=c \lambda
$$

with corresponding ghost and anti-ghost $c$ and $b$ satisfying $\{c, b\}=1$.

The gauge-fixing term in this case is

$$
\mathcal{L}_{g f}=\{Q, \Lambda\}
$$

where $\Lambda=b \Phi$ is the gauge-fixing function. The extra term $Q_{\text {extra }}$ gives the contribution (47), and there are also Fadeev-Popov terms from $b\{Q, \Phi\}$ with contribution from the term originally present in the BRST charge, $\tilde{c} \Delta N$. By using the gauge condition this contribution is $v \tilde{c} b$. Thus, the ghosts decouple in this gauge.

Then, one proceeds as before, solving the gauge condition and constraint.

Quantization in this gauge can also be achieved in a Gupta-Bleuler approach, by imposing the gauge condition and constraint on physical states. For any pair of physical states $|\chi\rangle$ and $|\phi\rangle$, we require:

$$
\langle\chi|\Phi| \phi\rangle=0
$$

But (54) is satisfied if we impose the following condition on physical states:

$$
\Phi \mid \text { phys }\rangle=0,
$$

Therefore, (55) implies,

$$
\left.\left.\alpha_{1}^{1} \mid \text { phys }\right\rangle=v \mid \text { phys }\right\rangle,
$$


Using (56), the $\Delta N=0$ constraint can be rewritten. Again, at the quantum level, for any pair of physical states $|\chi\rangle$ and $|\phi\rangle$

$$
0=\langle\chi|\Delta N| \phi\rangle
$$

should hold. But it is enough to impose:

$$
\left.\left(v \alpha_{-1}^{1}+\sum_{m \geq 2} \alpha_{-m}^{1} \alpha_{m}^{1}+\sum_{n \geq 1} \alpha_{-n}^{j} \alpha_{n}^{j}-\widetilde{\alpha}_{-n}^{i} \widetilde{\alpha}_{n}^{i}\right) \mid \text { phys }\right\rangle=0
$$

\subsection{The state}

Let us now consider a state in the gauge of the previous subsection of the following form

$$
\left|\phi_{0}\right\rangle=\left|\phi_{0}\right\rangle_{L} \otimes|n\rangle_{R}
$$

where the left-moving factor is a coherent state built on a left vacuum, $|0\rangle_{L}$,

$$
\left|\phi_{0}\right\rangle_{L}=\mathrm{e}^{-i v \alpha_{-1}^{2}-i v^{*} \alpha_{1}^{2}}|0\rangle_{L}
$$

and the right-moving part is an eigenstate of $N_{R}$ with eigenvalue $2 v^{2}$. We also set the parameters $x^{i}$ and $p^{i}$ to zero, making the center of mass the origin of the coordinate system. Notice that $\alpha_{1}^{2}\left|\phi_{0}\right\rangle_{L}=-i v$ which implies that $\left\langle\phi_{0}\left|\alpha_{-1}^{1}\right| \phi_{0}\right\rangle_{L}=v$ upon using equation (50).

We can compute now the expectation value of the string coordinates in the normalized state $\left|\phi_{0}\right\rangle$ to find a stationary circular loop of radius $v l$ :

$$
\begin{aligned}
\left\langle X^{0}\right\rangle & =2 l \tau \sqrt{2|v|^{2}-1}, \\
\left\langle X^{1}\right\rangle & =\frac{i l}{2}\left\langle\phi_{0}\left|-\alpha_{-1}^{1} \mathrm{e}^{2 i(\tau-\sigma)}+v \mathrm{e}^{-2 i(\tau-\sigma)}\right| \phi_{0}\right\rangle, \\
& =v l \sin 2(\tau-\sigma), \\
\left\langle X^{2}\right\rangle & =\frac{i l}{2}\left\langle\phi_{0}\left|-\alpha_{-1}^{2} \mathrm{e}^{2 i(\tau-\sigma)}+\alpha_{1}^{2} \mathrm{e}^{-2 i(\tau-\sigma)}\right| \phi_{0}\right\rangle, \\
& =v l \cos 2(\tau-\sigma) .
\end{aligned}
$$

There is no contribution from the right-moving bosonic excitations to the expectation value because we are considering that this sector is in a $N_{R}$ eigenstate.

Before ending this subsection, let us note that the circular loop is just one possible coherent state constructed using this gauge. It is not difficult to see the generalization to other shapes. Take a left-moving component for the state of the form:

$$
\left|\phi_{0}\right\rangle=\mathrm{e}^{\mathcal{A}}|0\rangle_{L}, \quad \mathcal{A}=\sum_{\{i, m\} \neq\{1,1\}} u_{m i} \alpha_{-m}^{i}+u_{m i}^{*} \alpha_{m}^{i},
$$


$i=1, \cdots, 8$ and $m$ runs over positive integers. If the right-moving part of the state has level $N_{R}=n$, then $\left\langle X^{1}\right\rangle$ is real provided

$$
n-v^{2}=\sum_{\{i, m\} \neq\{1,1\}}\left|u_{m i}\right|^{2} .
$$

Choosing the parameters $v$ and $n$ appropriately one can build a loop of arbitrary shape in target space. As a trivial example, consider $n=v^{2}, u_{m i}=0$ to obtain a folded string along the $X^{1}$ axis.

\section{Conclusions}

The use of coherent states allows for the construction of semiclassical states in superstring theory that bear close resemblance to the classical solutions. These are of relevance in both studies of some macroscopic defects expected to arise in string theory descriptions of inflationary cosmology and towards a microscopic entropy counting of certain string configurations that correspond to classical supergravity solutions.

For the open string case we have shown that the coherent state reproduces the classical motion in the target spacetime while other 'perturbative' excitations have large oscillations (averaging to zero) around the center of mass position of the string. In the closed string case similar properties are found. Also, with the gauge-fixing of section 3 we obtained the microscopic description of a left-moving static loop supported by a right-moving worldsheet current; the analog of a classical superconducting vorton solution [15].

As a final remark let us mention that throughout the text we have used ten dimensional spacetime thinking about the bosonic part of a superstring, the modification to include the fermionic sector being straightforward. In particular, the state of subsection 3.3 could have fermionic right-moving excitations accounting for part or the whole of the level $N_{R}$ needed. The latter possibility leads to the loop completely stabilized by fermionic excitations [12] which has no classical gravitational radiation.

\section{Acknowledgments}

We would like to thank Jaume Garriga, Ken Olum, Alex Vilenkin and especially Roberto Emparan and Jorge Russo for illuminating discussions. AI is grateful to the organizers of the Simons Workshop at Stony Brook and Perimeter Institute for their hospitality while this work was in progress. The work of AI was supported by DOE Grant DE-FG0391ER40674. WS was supported in part by NSF Grant PHY-0354776.

\section{References}

[1] N. Jones, H. Stoica and S. H. H. Tye, JHEP 0207, 051 (2002). S. Sarangi and S. H. H. Tye, Phys. Lett. B 536, 185 (2002) N. T. Jones, H. Stoica and S. H. H. Tye, Phys. Lett. B 563, 6 (2003). 
[2] G. Dvali and A. Vilenkin, Phys. Rev. D 67, 046002 (2003). G. Dvali and A. Vilenkin, JCAP 0403, 010 (2004).

[3] E. J. Copeland, R. C. Myers and J. Polchinski, JHEP 0406, 013 (2004).

[4] J. Polchinski, AIP Conf. Proc. 743, 331 (2005) [arXiv:hep-th/0410082]. J. Polchinski, arXiv:hep-th/0412244.

[5] L. Susskind, arXiv:hep-th/9309145.

[6] A. Sen, Mod. Phys. Lett. A 10, 2081 (1995) [arXiv:hep-th/9504147].

[7] A. Strominger and C. Vafa, Phys. Lett. B 379, 99 (1996) [arXiv:hep-th/9601029].

[8] A. Dabholkar, Phys. Lett. B 402, 53 (1997) [arXiv:hep-th/9702050].

[9] G. Calucci, Nucl. Phys. B 287, 658 (1987).

[10] M. B. Green, J. H. Schwarz and E. Witten, "Superstring Theory," Vol. 1, Cambridge University Press (1987).

[11] A. L. Larsen and N. G. Sanchez, Phys. Rev. D 62, 046003 (2000) [arXiv:hepth/0001180].

[12] J. J. Blanco-Pillado and A. Iglesias, Phys. Lett. B 636, 207 (2006) [arXiv:hepth/0512057].

[13] P.A. Collins and R.W. Tucker, Phys. Lett. 64B (1976) 207; L. Brink, P. Di Vecchia, and P. Howe, Phys. Lett. 65B (1976) 471; S. Deser and B. Zumino, Phys. Lett. 65B (1976) 369.

[14] W. Siegel, "Introduction to string field theory," Adv. Ser. Math. Phys. 8, 1 (1988) [arXiv:hep-th/0107094].

[15] R. L. Davis and E. P. S. Shellard, Phys. Lett. B 209, 485 (1988). J. J. BlancoPillado, K. D. Olum and A. Vilenkin, Phys. Rev. D 63, 103513 (2001) [arXiv:astro$\mathrm{ph} / 0004410]$. 